Psychology of Language and Communication 2015, Vol. 19, No. 2

DE GRUYTER

DEEN

DOI: $10.1515 /$ plc-2015-0008

JOSHUA WILLIAMS, ISABELLE DARCY, SHARLENE NEWMAN

Indiana University, Bloomington

\author{
SECOND LANGUAGE WORKING MEMORY DEFICITS \\ AND PLASTICITY IN HEARING BIMODAL LEARNERS OF \\ SIGN LANGUAGE
}

Little is known about the acquisition of another language modality on second language (L2) working memory (WM) capacity. Differential indexing within the WM system based on language modality may explain differences in performance on WM tasks in sign and spoken language. We investigated the effect of language modality (sign versus spoken) on L2 WM capacity. Results indicated reduced L2 WM span relative to first language span for both L2 learners of Spanish and American Sign Language (ASL). Importantly, ASL learners had lower L2 WM spans than Spanish learners. Additionally, ASL learners increased their L2 WM spans as a function of proficiency, whereas Spanish learners did not. This pattern of results demonstrated that acquiring another language modality disadvantages ASL learners. We posited that this disadvantage arises out of an inability to correctly and efficiently allocate linguistic information to the visuospatial sketchpad due to L1-related indexing bias. Key words: American sign language, working memory, second language acquisition, bimodal bilingualism

\title{
Introduction
}

The ability to store, manipulate, and integrate linguistic information (i.e., working memory) has been said to be the hallmark of language comprehension (Daneman \& Carpenter, 1980). Much of the research on the interface between working memory and language abilities have been relegated to monolingual spoken language research or speakers of a spoken language learning another

Address for correspondence: Joshua Williams, Psychological and Brain Science, Indiana University, 1101 E. 10th Street, Bloomington, IN 47405, United States. E-mail: willjota@indiana.edu 
spoken language (i.e., unimodal bilinguals). Relatively recently, however, there has been a shift to further understand how language modality (i.e., signed vs. spoken) affects cognitive processing. Sign languages differ from spoken languages in how language is articulated. Sign languages use a visual-manual modality, whereas spoken languages use the auditory-oral modality. Learners who are acquiring a sign language as a second language (L2) when their first language is a spoken language (i.e., bimodal bilinguals, Emmorey et al., 2008) must learn how to adapt their already-instantiated system to process language in a new modality. As such, research is needed to determine which areas of cognition are affected (or require adaptation) by a new language modality. The present study aims to investigate how working memory (WM) is differentially affected for unimodal Spanish learners and bimodal American Sign Language L2 learners. We specifically aim to test four hypotheses: whether 1) WM span in the first language (L1) is larger than their L2 span for both unimodal and bimodal learners; 2) bimodal L2 learners will have a significantly worse L2 WM span relative to unimodal L2 learners; 3) traditional phonological WM measures (e.g., backward digit span) will correlate with L2 WM span for the unimodal L2 learners; and lastly, whether 4) greater language exposure, or proficiency, will allow the bimodal L2 learners to improve their WM span, approximating that of the unimodal L2 learners.

\section{Working memory}

The temporary storage and manipulation of information resides in working memory (Baddeley \& Hitch, 1974). According to their model, working memory is fractionated into separate components that facilitate performance across a range of cognitive tasks. The phonological loop contains a temporary verbal-acoustic storage system that facilitates the retention of sequences (e.g., digits, letters, words, etc.). The visuospatial sketchpad is another subsystem that integrates spatial, visual, and kinesthetic information into a unified representation to be stored and manipulated within working memory. Finally, the central executive is responsible for the attentional control of the working memory system. Baddeley (2000) later added a fourth component, the episodic buffer, which is responsible for the combining of multiple information types into a single multi-modal, multifaceted representation. Among the several components of the working memory system, it has often been thought that language processing is impacted primarily by the phonological loop (Baddeley, 2003).

The phonological loop can be described in terms of both its storage capacity and storage quality. The storage capacity is often measured with serial order recall tasks (e.g., digit span tasks or complex span tasks). There is debate regarding the amount of information that can be temporally stored in working memory (Miller, 1994; Cowan, 2001); nevertheless, there is widespread agreement that working memory storage capacity is limited (Baddeley, 2000; Cowan, 2001). For the purposes of this study, storage quality is defined by the quality of the phono- 
logical representation that is stored. Storage quality is indexed by performance differences in memory tasks conditioned by phonological knowledge, such as typicality or word-likeness effects (Gathercole \& Baddeley, 1990). The quality of a phonological representation, thus, impacts capacity insofar as lower quality representations reduce capacity (see Oakhill \& Kyle, 2000 for evidence of decreased phonological awareness skills and working memory). This has implications on language comprehension and production, especially for second language learners who do not have well-specified representations (Broselow \& Finer, 1991).

\section{WM and language}

The phonological loop has been implicated in language comprehension and acquisition. Vallar and Papagno (2002) propose a model in which the phonological buffer is required for comprehension and production of spoken language. Studies of first language acquisition have posited that impairments in the phonological storage component of the loop predicts decreases in language performance, as evidenced by children with specific language impairment who perform poorly on nonword repetition tasks despite normal articulatory and auditory abilities (Gathercole \& Baddeley, 1989). Taken together, the working memory system, especially the phonological loop and its components, have been shown to underlie language ability.

Daneman and Carpenter (1980) hypothesized that individual differences in native language abilities were borne out of the variability in working memory systems across individuals. Daneman and Carpenter posited that the functional capacity of the working memory system would directly predict language abilities. The correlation between working memory capacity and language is thought to arise out of the limited resources being shared across processing and storage demands within the working memory system. Specifically, functionally smaller storage capacities lead to deficits in comprehension because the ability to integrate successive information is impaired (Daneman \& Merikle, 1996). Reading and listening span tasks, which were thought to tax both the processing and storage components, were implemented to test this hypothesis. The authors found that individuals with smaller reading/listening spans performed worse on language tests than those with higher spans (Daneman \& Carpenter, 1980). The connection between individual differences in working memory has since been replicated and extended across cognitive domains (e.g., visual working memory, Vogel \& Machizawa, 2004; see Daneman \& Merikle, 1996 for a review).

\section{WM and $L 2$ acquisition}

Not only does working memory affect native language processing, but it also has implications on second language processing. Moreover, it is important to understand why working memory capacity differs between a learner's first and second language. Second language learners' target-language digit spans are smaller than their first language spans (Ardila, 2003). Nevertheless, increased 
working memory capacity is correlated with greater subsequent language acquisition and aptitude. Increased memory task performance (e.g., digit span), but not intelligence or visuospatial memory, is associated with increased ability to learn new words in a foreign language (Papagno \& Vallar, 1995). Specifically, auditory phonological working memory has been implicated in the ability to learn a new language (Baddeley, Papagno, \& Vallar, 1988). In fact, some have argued that auditory working memory capacity serves as a predictor for second language proficiency (Miyake \& Friedman, 1998). A possible suppression mechanism has been posited to explain the correlation between working memory capacity and second language proficiency. That is, storage capacity is linked to more efficient suppression of intrusive thoughts or behaviors (Rosen \& Engle, 1998) and as such a larger storage capacity (or a better span) could help learners suppress the influence of L1 during L2 processing. With evidence that English-ASL bilinguals do not need to suppress their languages similarly to unimodal bilinguals (e.g., English-Spanish bilinguals; Emmorey, Luk, Pyers, \& Bialystok, 2008; Williams \& Newman, 2015), one could posit that there would be a de-correlation between digit span and L2 acquisition for sign language. In other words, working memory capacity may only be predictive for spoken language acquisition.

Differences across first and second language working memory capacities do not imply that learners have separate memory systems for each language. An integrative view of second language working memory should be adopted and is motivated by previous research. For highly proficient bilinguals, first and second language reading spans are often correlated (Osaka \& Osaka, 1992; Osaka, Osaka, \& Goner, 1993). Additionally, proficiency in both native and nonnative languages is correlated (Cummins, 1991; Carson, Carrel, Silberstein, Kroll, \& Kuehn, 1990). In other words, the capacity of an individual's working memory in their first language will predict storage and manipulation capacity in their second language. The differences in working memory performance between languages are not due to the structure of a separate system per se, but rather the quality of the code within working memory. As a result, because the input quality is less "pristine" (e.g., similar to native language quality), the overall storage performance and manipulation of that input is subject to faster decay. The ability to maintain and manipulate underspecified information could account for correlations between stronger first and second language working memory (Bays \& Husain, 2008). If one takes the integrative approach, the question remains how the storage and manipulation of different language modalities affect the working memory system.

\section{WM, language, and modality}

Working memory components, like the phonological loop, are vital to language processing. Given that the phonological loop is often conceptualized as a verbal memory storage, it is of some theoretical importance to understand how individuals with a visual language store and manipulate information in working 
memory. Sign languages, such as American Sign Language (ASL), are produced in the manual-visual modality, whereas spoken languages like English and Spanish are produced in the oral-auditory modality (barring orthography and reading). The phonological loop is well conceptualized for spoken languages, but how does the phonological loop operate when processing visual languages?

Several studies have outlined the working memory system in native deaf signers (Wilson \& Emmorey, 1997a, b, 1998; Boutla, Supalla, Newport, \& Bavelier, 2004; Hall \& Bavelier, 2010; Hirshorn, Fernandez, \& Bavelier, 2012). Wilson and Emmorey (1997a,b, and 1998) demonstrated that native deaf signers show similar phonological effects (i.e., phonological similarity effect, word length effect, articulatory suppression effect) for sign language. Native deaf ASL signers have significantly lower digit spans (i.e., $5 \pm 1$ ) than typical native hearing English speakers (i.e., $7 \pm 2$ ). These differences cannot be accounted for by speed of articulation or rehearsal (Boutla et al., 2004; Hall \& Bavelier, 2010) or the differences in the number of phonological parameters in a given sign (Hirshorn et al., 2012). It is thought that experience with auditory language and its serial properties create the environment to induce better temporal/phonological working memory systems for hearing individuals (Conway, Pisoni, \& Kronenberger, 2009). That is, phonological working memory relies on temporal processing and is correlated with language rehearsal, but not visual rehearsal (Saito, 2001). Alternatively, while sign languages have temporal features, the heavy reliance on simultaneous visual language processing affords deaf signers better spatial working memory abilities (Wilson, Bettger, Niculae, \& Kilma, 1997). The inherent differences in the languages themselves, and in the processing strategies they require, call for differential use of the working memory system.

Differential use of the working memory system may mean that various subcomponents are preferentially allocated for each language modality. As mentioned above, the visuospatial sketchpad integrates visual and spatial information into a single code. Thus, the differences in language modalities might influence the preference of deaf signers' use of the visuospatial sketchpad for spatiotemporal indexing and hearing speakers' use of the phonological loop for temporally ordered information (Hirshorn et al., 2012). Hirshorn et al. posited that there is a bias in the use of a specific slave system (i.e., indexing strategy) during language processing and rehearsal. That is, language users differentially allocate processing to specific buffers depending on the spatiotemporal or phonological characteristics of the representation entering into working memory. For example, the authors tested three conditions: spatiotemporal, phonological, and spatiotemporal and phonological. They posited that deaf signers would outperform hearing nonsigners on the spatiotemporal task, the hearing nonsigners would outperform deaf signers on the phonological task, and when both cues were available their performance would be matched. In fact, this is what the authors found. In other words, there is relative biasing for which memory subcomponent is used dur- 
ing language processing based on its modality. Relative biasing and indexing strategies can account for differences in span lengths across deaf and hearing populations. This hypothesis may prove to be important when examining second language learners of a different language modality in terms of their reallocation of phonological memory processing.

\section{WM, L2 acquisition, and modality}

The affordances of a given language modality alter working memory taskrelated effects. The differences between hearing and deaf individuals in terms of decreased digit span as well as increased spatial working memory performance have also been demonstrated in nonnative learners of sign (Capirci, Cattani, Rossini, \& Volterra, 1998; Keehner \& Gathercole, 2007). Furthermore, language modality has been shown to bias individuals towards the use of differential working memory subcomponents (Hirshorn et al., 2012). Second language learners who are learning another language within the same modality (e.g., EnglishSpanish learners) could show similar working memory capacities barring any quality issues. On the other hand, second language learners who are learning another language and language modality (e.g., English-ASL learners) could pattern similarly to deaf participants. The hypothesis tested here is that a difference in language modality between the first and second language will have greater negative effects on second language working memory than when the first and second language have the same modality.

\section{Predictions}

In this study, a listening span task was implemented in order to investigate differences in working memory span for the first and the second language. Additionally, the differences between first and second language working memory spans were examined by changing the language modality of the second language. A listening span task involves participants listening to a sentence and remembering the last word in each sentence. At the end of a given number of sentences the participant is asked to recall the last words of each sentence in the order in which they were presented (see Daneman \& Carpenter, 1980). In the present study, the listening span tasks for English and Spanish were similar to what has been reported previously. In regards to ASL, the learners watched videos of ASL sentences and recalled the last sign in each sentence. Thus, the use of "listening span" in the present study refers to normal modes of receptive comprehension for each respective language modality. A listening span task can, therefore, be implemented in both language modalities in order to capture working memory spans. Using this task, the following predictions were made:

1. Given that previous research has shown that L1 working memory span is better than in L2 (Ardila, 2003), it was predicted that the first language listening span in English would be significantly larger than the second 
language listening spans in Spanish and ASL for both unimodal Spanish and bimodal ASL learners, respectively. Increased first language span was posited to not only be due to language-specific differences, but also the quality of the representations. By testing this hypothesis, we can provide a replication of previous literature to add validity to our own experiment, but also provide additional evidence about working memory capacities across L1 and L2 that are modality-independent.

2. Given that ASL learners are exposing their working memory system to a new type of language modality, either incorrectly into the phonological loop or the less-conditioned episodic buffer and/or visuospatial sketchpad, it was predicted that their L2 listening span would be significantly smaller than Spanish learners' L2 span. That is, it is hypothesized that second language spans will be smaller for ASL learners compared to Spanish learners due to the language modality differentially biasing the subcomponent use and differences in code familiarity. Conversely, the Spanish students who have experience with a spoken code will demonstrate fewer differences between L1 and L2 span length based on the use of a practiced phonological loop. Within-modality similarities and across-modality differences in L1 and L2 spans provides great insights into how language modality (or code similarity) is related to L2 acquisition.

3. Given that the similarity of language modality is important to the predictive power of traditional working memory measures (i.e., digit span) on L2 acquisition, it was hypothesized that digit span will only predict L2 spans for Spanish students, not ASL learners, because of language modality differences. Since the digits are sequentially stored and processed and within the same spoken modality, digit span will tap into the same phonological loop as Spanish; however, this will not be the case for ASL, which likely uses the visuospatial component. The differential effects of language modality on working memory span were examined using correlational analyses. A positive correlation between digit span and L2 span for Spanish learners, but not ASL learners, is important to our ability to locate how differential indexing in working memory impacts L2 comprehension across language modalities.

4. Given that we have argued that language experience impacts ASL learners' ability to correctly index information into working memory, it was hypothesized that there would be a concomitant increase in listening span as proficiency increases. However, it was expected that only the ASL students, who are acquiring a different code, would show a strong correlation between listening span and proficiency. Spanish students may have an increase in their L2 working memory because they have better phonological coding abilities for rehearsal, but a marked gain should be mostly visible in the group with a different language code. The investigation 
of interlanguage dynamics, or how cognitive processes are modulated by L2 proficiency, on working memory is important for understanding how individual differences influence acquisition of a second language (Williams \& Newman, 2015).

Taken together, the present study was designed to provide deeper insight into how language modality affects working memory in L2 learners. This study advances the state-of-the-art in second language acquisition by positing that learners who are acquiring a sign language as a second language must learn how to appropriately allocate visuospatial linguistic information from the phonological loop to the visuospatial buffer.

\section{Method}

\section{Participants}

Forty students from Indiana University participated in this study. Two groups of students were recruited based on their second language. Twenty students were low- to high-intermediate Spanish students who were currently enrolled or had taken $3^{\text {rd }}$ and $4^{\text {th }}$ semester Spanish courses. Similarly, there were 20 students who were currently enrolled in $3^{\text {rd }}$ and $4^{\text {th }}$ semester American Sign Language courses. All of the students completed a background questionnaire that collected individual proficiency self-ratings on a scale including "very poor," "fair," "functional," "good," "very good," and " near native" in speaking, understanding, reading, and writing. In the case of ASL students, their reading and writing scores were not factored in as ASL does not have an official, commonly used writing system. Self-ratings have been shown to correlate with measured proficiency (MacIntyre, Noels, \& Clement, 1997; Bachman \& Palmer, 1989). Proficiency was also measured using language-specific assessments. The Spanish students took a translated version of Peabody Picture Vocabulary Test (Padilla, Lugo, \& Dunn, 1986). The ASL students took a Fingerspelling Reproduction Test (FRT; Visual Learning and Visual Language Center, Gallaudet University, Washington, D.C.; Morere, 2008). Given the relative scarcity of standardized ASL measures, the FRT was chosen because it was a rather accessible measure that had been used previously with deaf signers and is shown to correlate with ASL comprehension (Hauser, Paludneviciene, Supalla, \& Bavelier, 2006; Mayberry \& Eichen, 1991). In order to normalize scores that allow us to have a composite measure of individual proficiency as well as to provide between-group comparisons, composite proficiency scores were calculated by taking a ratio of percent correct of raw scores on the individual measures and their self-rating. The proficiency scores ranged from 0 to 1 (and can be converted to percentages, if desired). A composite of 0 would indicate a naïve learner, 0.5 would indicate an intermediate learner, and a 1 would indicate a native-like learner. Composite proficiency scores for the Spanish students ranged from 0.339 to $0.736(\mathrm{M}=0.597, \mathrm{SD}=0.098)$. Composite proficiency scores for the 
ASL students ranged from 0.364 to $0.850(\mathrm{M}=0.558, \mathrm{SD}=0.116)$. The composite scores did not significantly differ between groups, $F(1,39)=2.778, p>0.1$. We posit that these are good measures of proficiency in our groups because they take into account actual L2-specific skills and self-rating. Furthermore, the proficiency score correlates well with duration of L2-use $(r=0.636, p<0.001)$ by both groups of learners. That suggests that this score measures proficiency as a function of amount of input and learning. Although it could be argued that this composite proficiency score measures different constructs across the languages (ASL: sub-lexical knowledge; Spanish: lexical-semantic knowledge), analyses will be carried out with both composite and self-rating scores since self-rating is 1) a valid measure of proficiency, 2) the same across groups, and 3) correlated with their composite score $(r=0.889 ; p<0.001)$.

The forward and backward English digit spans of the participants were also collected. A digit span task was included in order to capture another verbal working memory measure that has been shown to be a predictor for L2 language competence (see Ardila, 2003). Furthermore, we hypothesized that L1 digit span is only predictive of L2 working memory ability when the L1 and L2 share the same language modality. Thus, this hypothesis can be directly tested by examining correlations between digit span and L2 listening span. Any positive evidence to this hypothesis would also support the claims that ASL learners must use a visuospatial buffer rather than a verbal/phonological buffer.

\section{Speakers / signers}

Three native speakers of the target languages recorded the stimuli. The English speaker was a 24-year-old male native speaker from the United States who reported learning Spanish and ASL in his late teens. English was his dominant language. The Spanish speaker was a 35-year-old male native speaker from Castilian Spanish. He also speaks French, German, Haitian, Portuguese, and English. However, Spanish was his dominant language. The ASL signer was a 21-year-old male hearing bimodal bilingual. The bimodal bilingual was born hearing to deaf parents (CODA). He reported that his first language was ASL and his second language was English, although they were learned at relatively the same time. English was his dominant language, but he reported to still use ASL every day.

\section{Stimuli}

The task was a Listening Span Task (LST; Daneman \& Carpenter, 1980) with a final word translation task. The stimulus set consisted of 58 sentences. The sentences were videos of a native language user either speaking or signing the stimuli. The presentation mode controlled the amount of modality input so that results were not confounded by audio-only and visual-only conditions. The sentences were selected from three sources: Pisoni, Manous, \& Denina (1987), Speed and Capacity of Language Processing Test (SCOLP; Baddeley, Emslie, \& Nimmo- 
Smith, 1992), and the Alloway Working Memory Assessment 2 (AWMA; Alloway, 2007). The sentences were equally split into plausible (e.g., "Most people like to receive love.") and implausible (e.g., "Fathers are younger than their daughters.") sentences. The 58 sentences in Spanish and ASL corresponded to the 58 English sentences. Spanish and ASL sentences were translated by the experimenters and validated by another native speaker/signer. For each language, there was a practice block and subsequently two blocks per length, ranging from 2-sentence sets to 7 -sentences sets. Each block was constructed by randomizing the sentences and assigning sentences to each block. Randomization was done for each of the languages, as the sentences for all of the languages were identical.

Digit span task was implemented in the learners' first language, English. The numbers were pseudo-randomly selected during stimulus design such that no sequence had the same digit twice. All participants heard the same sequence of numbers. There were two lists: forward and backward. Both lists contained different sequences of numbers. There were two trials of the same sequence length, starting at 2 and increased by one until 9 .

\section{Procedure}

The students performed both English and the respective L2 LST. Following the traditional LST paradigm (Daneman \& Carpenter, 1980), the students saw a sentence and were asked to judge whether it was plausible or implausible as quickly as they could. At the end of the plausibility judgments for all of the sentences within a set, the students were asked to recall the last word of each sentence as they were presented by typing in the English translation. For the English LST, participants had to provide the last word in the sentence in English. For the L2 LST, participants had to translate the last word from their L2 into English. A translation procedure was adapted in order to equate the task across the languages (i.e., regardless of the L2 modality, both groups had to respond in their L1). Additionally, given that there may be greater production variability in L2 ASL learners (see Hilger, Loucks, Quinto-Pozos, \& Dye, 2015) and we were only interested in modality effects on comprehension, we did not want the production of the L2 words/signs to mar the subjects' accuracy, especially with potential differences in production abilities across the two L2 learner groups.

The students saw a practice set with 4 sentences. After the practice, there were two blocks of the same number of sentences, increasing from 2 sentences to 7 sentences in a set. Regardless of the answers, all blocks were administered. Since the sentences were similar across the languages, the language order was counterbalanced across subjects. For example, the first student would perform the English LST, then their respective language proficiency tests, and finally their L2 LST. The next student would perform the L2 LST, the proficiency tests, and finally the English LST. This was to wash out an ordering effect. Moreover, to reduce a potential effect of language, the proficiency tests were interspersed 
between LSTs to minimize remembering from one LST to the other. The sentences were pseudo-randomized across languages in order to prevent the same sentence from appearing in the same set across languages. There are three measures for each LST task: a recall score, a plausibility score, and processing time. The recall scores were calculated only if all the words in a given set were recalled in the correct serial order, but regardless of plausibility rating. Scoring was based on correct word report judged by the Experimenter barring any spelling errors in at least one of the two sets of the same length. That is, if the participant recalled all the words correct in both sets at a given length (e.g., $l=2)$ but did not correctly recall all the words in either of the sets within the next length (e.g., $l+1=3$ ), then their score would be the previous correct set (e.g., 2). Similarly, if the participants recalled all the words in both sets correct at a given length (e.g., $l=2$ ) and also recalled all the words in one of the two sets of the next length (e.g., $l+1=3$ ), then their score would be of that length (e.g., 3). Processing times were calculated only for the sentences for which plausibility responses were correct.

The procedure for digit span measures was similar to the listening span task. The learner's were presented a list of numbers aurally at about one digit per second. For the forward condition, the participants were to recall the sequence of numbers in the correct order in which they heard them. For the backwards condition, the participants recalled the sequence of numbers in the reverse order. Span was scored based on the same criterion as above.

\section{Results}

\section{Listening span}

A 2 (language: L1 vs L2) by 2 (learner: Spanish vs. ASL) analysis of variance (ANOVA) of the listening spans was performed by subject to investigate differences between the first and second language listening spans across groups. There

Table 1. Presents the descriptive statistics for each measure in each language

\begin{tabular}{lcccc}
\hline & \multicolumn{2}{c}{ Spanish } & \multicolumn{2}{c}{ ASL } \\
\hline \multicolumn{1}{c}{ Measure } & L1 & L2 & L1 & L2 \\
\hline Proficiency & & 0.61 & & 0.56 \\
LST Recall Score & 4.75 & 3.05 & 4.8 & 2.45 \\
LST Plausibility Score & $98.20 \%$ & $75.10 \%$ & $98.30 \%$ & $56.70 \%$ \\
LST Processing Time (s) & 4.41 & 7.59 & 4.45 & 7.29 \\
Forward Digit Span & 6.5 & & 6.5 & \\
Backward Digit Span & 4 & & 4.2 & \\
\hline
\end{tabular}


was a significant main effect of language $\left(F_{(1,38)}=190.319, p<0.001, \eta_{p}^{2}=0.834\right)$. There was also a significant interaction effect of language across the learner groups $\left(F_{(1,38)}=4.902, p<0.05, \eta_{p}^{2}=0.114\right)$. Simple effects analyses were run to understand the differences within the condition. There was no effect of learner group in English (L1) listening span, $F_{(1,38)}=0.035, p=0.852$. However, there was a significant effect of learner group in the second language (L2) LS, $F_{(1,38)}=6.874, p<0.05$. The Spanish students had a significantly higher $\mathrm{L} 2$ listening span $(\mathrm{M}=3.05, \mathrm{SD}=0.759)$ than ASL students $(M=2.450, S D=0.776)$. This cannot be attributed to group proficiency differences since both groups were similar in proficiency (see Method section).

\section{Plausibility}

A similar 2 by 2 ANOVA (language by learner) was performed to investigate differences in the accuracy of plausibility judgments. There was a significant main effect of language $\left(F_{(1,38)}=546.841, p<0.001, \eta_{p}^{2}=0.935\right)$. The interaction effect of language with learner group was also significant $\left(F_{(1,38)}=44.429, p<0.001\right.$, $\eta_{p}^{2}=0.539$ ). One-way ANOVAs were ran to understand the differences within the condition. The accuracy of plausibility judgments did not differ across learner groups for their first language, $F<1$. The accuracy on the L2-LST did in fact differ across groups $\left(F_{(1,38)}=41.245, p<0.001\right)$, where the Spanish students were more accurate $(M=0.751, S D=0.078)$ than the ASL students $(M=0.567, S D=0.101)$.

\section{Processing times}

Another $2 \times 2$ ANOVA with the same within-subject factor of language and between-subject factor of learnergroup was performed to investigate differences between the processing times of plausibility judgments. There was a main effect of language $\left(F_{(1,38)}=115.950, p<0.001, \eta_{p}^{2}=0.753\right)$, such that all learners were significantly faster for their $\mathrm{L} 1(\mathrm{M}=4.427, \mathrm{SD}=0.234)$ than their $\mathrm{L} 2(\mathrm{M}=7.444$, $\mathrm{SD}=0.372)$. However, there was no interaction with learner group, $F<1$.

\section{Ad-hoc correlations}

Ad-hoc correlation analyses were performed in order to investigate the effects of proficiency on listening span as well as the ability for the digit span measure to predict L2 listening span. There was a trending increase in L2 LS as a function of L2 proficiency for ASL students $\left(r=0.386, p=0.093, R^{2}=0.149\right)$ but not for Spanish students $\left(r=0.053, p=0.825, R^{2}=0.003\right)$, see Figure 2. It could be argued that the composite proficiency score is not equal across groups since it takes into account different tests across the language groups. A secondary correlation analysis with L2 LS and L2 self-report showed similar results (ASL: $r=0.389, p=0.090, R^{2}=0.152$; Spanish: $r=-0.094, p=0.695, R^{2}=0.009$ ).

We investigated the influence of proficiency. There was a significant negative correlation between proficiency and differences in L1 and L2 spans for ASL students $(r=-0.554, p<0.01)$ but not Spanish students $(r=-0.083, p=0.729)$. 
Figure 1. Contains two regression plots correlating composite proficiency scores and second language listening span for the ASL (left) and Spanish (right) learners. There is a positive correlation of proficiency on span for ASL students, but not Spanish
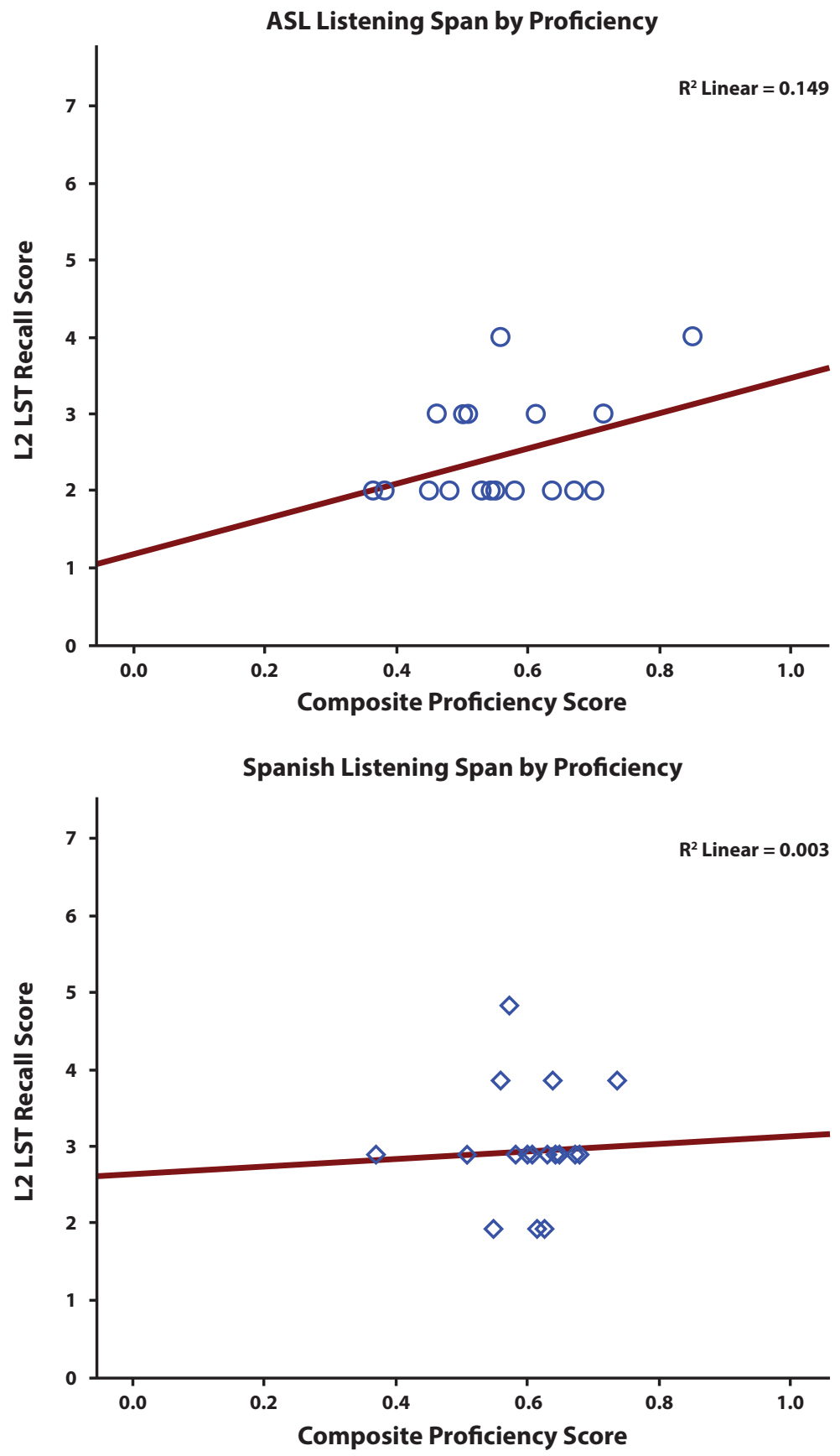
Figure 2. Shows that the difference between L1 and L2 spans changes as a function of proficiency for ASL learners (left), but not Spanish learners (right). This indicates that as the ASL learners become more proficient, their L2 span approximates their L1
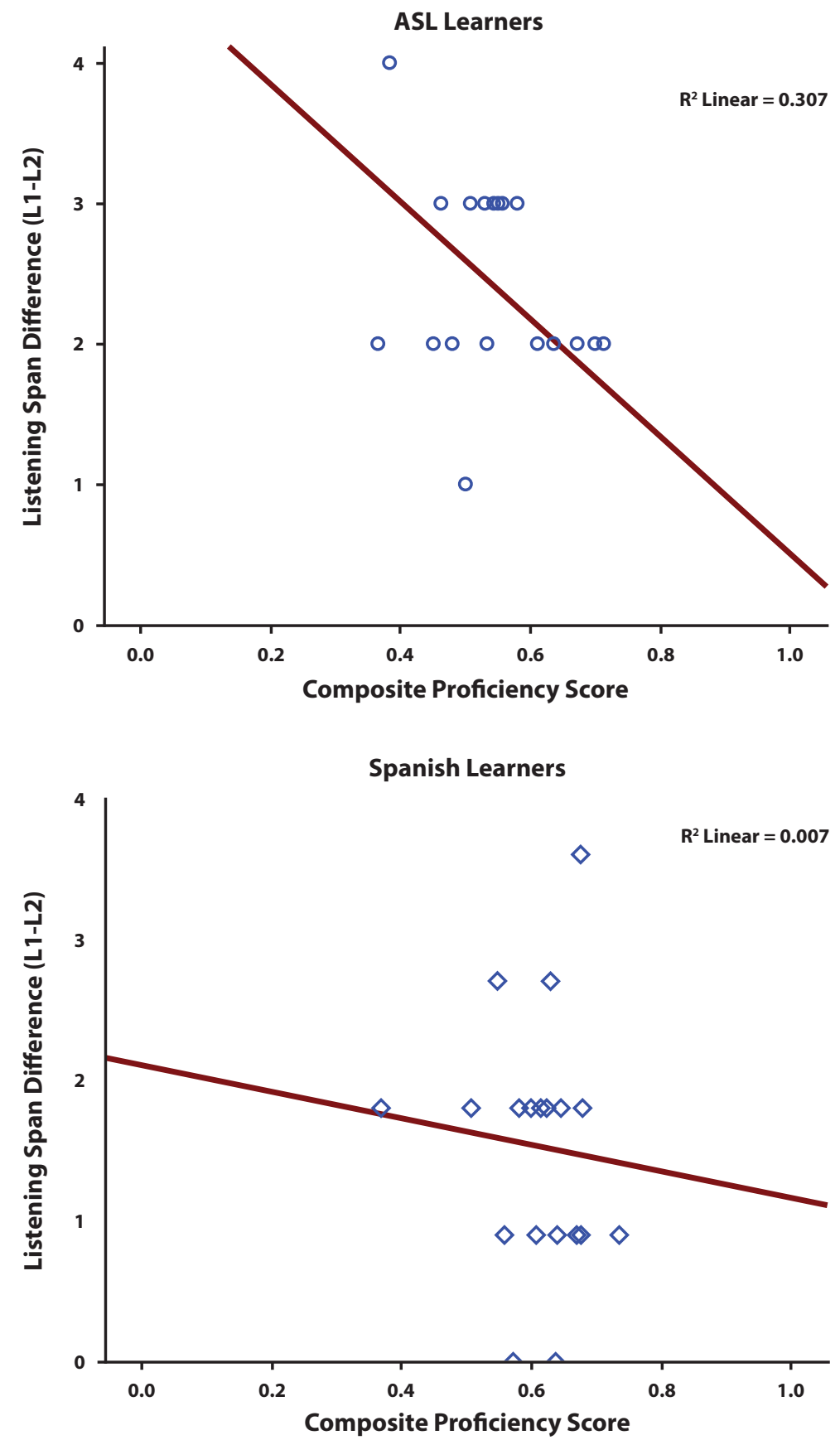
Figure 2 indicates that proficiency accounts for much of the difference $\left(R^{2}=0.307\right)$ for ASL students. Similarly, the results were the same when self-reported proficiency was taken into account (ASL: $r=-0.532, p=0.016, R^{2}=0.283$; Spanish: $\left.r=-0.012, p=0.959, R^{2}=0.000\right)$.

The relationship of traditional memory measures with the L1 and L2 spans was explored through the correlation between forward and backward digit span. There was a positive correlation between the forward digit span and the L2 listening span for Spanish learners $(r=0.494, p=0.027)$ but not for ASL $(r=0.076$, $p=0.750)$. The backward digit span did not correlate with either group.

\section{General discussion}

The goal of the current study was to investigate the role effect of language modality on working memory capacity of second language learners and how interlanguage dynamics reallocates processing in working memory based on language modality. This study extends our understanding of the interaction between working memory and language modality in a number of ways. First, this study demonstrated a reliable difference in listening span between groups acquiring a second language in a different modality. Furthermore, there are differential working memory effects between first and second language spans as a function of language modality and proficiency. Lastly, traditional memory capacity measures predict same-modality spoken second language listening span, but not different-modality listening span.

\section{L1 vs. L2 working memory}

The present study used a listening span task in order to investigate working memory capacity for both first and second languages. The listening span task allowed for an investigation into working memory during sign or spoken language comprehension, which accommodated the fact that there is no widely used written system in ASL. Also, the span task taxed and engaged the working memory system to drive maximal differences across language modalities. Our results showed the amount of L2 information that can be recalled from working memory is less than L1 linguistic information. A decreased L2 span compared to L1 comes as no surprise as the quality of the L2 representations are thought to decay more quickly within memory (Miyake \& Friedman, 1998). Therefore, despite differences in language modality, learners are less able to store and manipulate second language representations in working memory compared to their first language.

\section{WM and language modality}

The role of different language modalities is important to theories of working memory in second language acquisition as well as working memory in language processing in general. This study demonstrated that the ability to encode and 
retrieve linguistic information in working memory differs based on language modality. Although this has been shown in previous studies that investigated working memory in native deaf signers, this is the first study to investigate the role of language modality in second language acquisition. Examining second language learners' performance allows for an investigation into differences in a working memory system that is already attuned to a specific code and indexing strategy. According to Hirshorn et al. (2012), hearing speakers have a preference (i.e., relative bias) for using the phonological loop to process spoken languages, whereas deaf signers have a preference for the visuospatial sketchpad. These biases arise out of the nature of the language modality itself. In the case of hearing ASL learners, their system is already biased to a highly temporal, spoken-language code typically processed in the phonological loop. We hypothesized that their phonological working memory is better than their spatial working memory (see Emmorey, Kosslyn, \& Bellugi, 1993 for ASL advantage in spatial working memory tasks). Thus during second language acquisition, or the acquisition of another code, their working memory system has to accommodate these changes. Indeed, the hypothesis was confirmed. ASL learners had significantly lower listening spans than their Spanish learner counterparts.

Furthermore, the difference between first and second language spans was significantly reduced with increased proficiency for the ASL students, but not the Spanish students, which indicates that ASL students might learn how to manipulate visuospatial linguistic information over time. That is, the stable spans for Spanish students across proficiency levels may be attributed to their L1 expertise in phonological processing, whereas ASL learners had to learn to manipulate the visual signs over time, slowly increasing the amount of information that can be stored, manipulated, and/or recalled from working memory.

It is tempting to attribute differences between ASL and Spanish L2 spans to general span differences across language modalities [e.g., native deaf ASL signers have significantly lower digit spans (i.e., $5 \pm 1$ ) than typical hearing native English speakers (i.e., $7 \pm 2$ )]. However, this may not be the case. Previous research has shown that the mean digit span for native Spanish speakers is 5.8 (Ardila, 2003) and for native deaf signers is $5 \pm 1$ (Morere \& Allen, 2012). Therefore, the native benchmark in this study is relatively the same for both learner groups. Spanish learners were able to better approximate their native benchmark, whereas the ASL students were not. We argue that the differences between ASL and Spanish L2 span is not due to a difference in proportional native benchmarks, but rather to the possibility that ASL students are trying to use the phonological loop to processes visuospatial information or, more probably, that they have less experience using the visuospatial sketchpad to store linguistic information.

Here it was hypothesized that signed and auditory languages are biased to use visuospatial and phonological working memory systems, respectively. This differential use of these two WM systems, along with the fact that the capacities 
of these systems are not necessarily correlated (Park et al., 2002) may account for differences in the relationship between L1 and L2 span observed (see Figure 2). In other words, a possible explanation for an ASL learner who has a small L1 span but a high L2 span may be that they have poor phonological working memory but enhanced visuospatial working memory. Conversely, an ASL learner who has a large L1 span but a low L2 span may have poor visuospatial working memory. However, Spanish learners are not hypothesized to have these differential working memory effects, as their L1 and L2 lie within similar modalities. This means that the differences in modality necessitates differential indexing strategies: allocation to either phonological or visuospatial buffers. Although this is unknown from the present study, as we did not collect spatial working memory measures, such a mechanism would provide an account for these differential working memory effects. Differential effects seem to arise from the extant system and how relative bias is preferentially allocated. The preferential indexing could be a 'learned' process that increases with proficiency.

As an alternative strategy, driven by the demands of the translation task, all of these students may be immediately recoding from their second language to their first language in order to maintain and rehearse the last word of the sentence in the phonological loop. Thus, the conversion of phonologically similar languages (i.e., Spanish to English) does not hinder the working memory system relative to the first language as much as the converting from phonologically distinct systems (i.e., ASL to English). Nevertheless, this strategy does not preclude or dissociate itself from inefficient allocation of the code to the appropriate slave system. The deficits derived from recoding (or translating) can be attributed to the hearing ASL learners having a bias towards sending the initial ASL information to the inappropriate slave system. Thus, whether the learners are rehearsing the last word/sign of the sentence in their L1 or their L2, the initial allocation within the memory system is influenced by the learners' bias, leading to initial or long-term indexing errors. Indexing errors (i.e., sending information to the wrong slave system) can ultimately account for differences between the ASL and Spanish span lengths.

\section{WM, modality, and L2 proficiency}

The fact that the L2 listening spans of more proficient ASL learners started to approximate the L2 span of the Spanish students suggests that the system can overcome modality deficits and increase the ability to store and manipulate visuospatial information, as well as recoding it into verbal information. This indicates a greater plasticity in indexing strategy as a function of proficiency. This could be important to increased grammatical competence in ASL L2 learners, as they can increase the amount of information that can be allocated to working memory. Although learners may be able to reallocate linguistic information to specialized, modality-specific subcomponents, there could be limitations on 
the approximation to L1 processing. That is, the ASL learners' spans may still plateau at a length around 5 due to the constraints seen in deaf signers, similar for the Spanish learners and the native Spanish constraints. Nevertheless, the ability to better allocate information into the working memory system for L2 sign language provides a future avenue for research into training working memory to facilitate language learning. It should be noted that we are not claiming that absolute working memory capacity is changing as a function of proficiency or language modality; rather, the ability to index appropriate phonological or visuospatial information into the corresponding working buffers improves with language exposure and competence.

On a practical note, the correlation between L1 digit span and Spanish L2 listening span $(r=0.494)$ is indicative that digit span measures spoken-language specific capacity over that of signed language. Thus, a connection between tasks and cognitive outcomes has an important impact on the measurement of working memory in ASL L2 research. The modality affords more spatiotemporal processing, and thus any ASL L2 research that requires a cognitive measure of memory must accommodate visual language affordance by administering spatial memory tasks (e.g., Corsi block test). As mentioned in Hirshorn et al. (2012), the differences in language modality and in the types of results seen across tasks need to be explicitly accounted for by the mechanisms that are theorized to underlie the task. Language-specific and task-specific manipulations may provide further insight into the structure and function of the working memory system.

\section{Conclusion}

In conclusion, this study provides the first account of how language modality affects working memory capacity for different groups of second language learners. This study also provides reasoning to change how L2 working memory in ASL students is measured for research purposes, as digit span may be testing another construct. More importantly, this study shows that learners may be able to change how specific information is allocated to a given working memory based on a specific language modality, and the deficit induced by learning a second language modality can be overcome to approximate that of second language learners.

\section{References}

Alloway, T. P. (2007). Automated Working: Memory Assessment: Manual. London: Pearson Assessment.

Ardila, A. (2003). Language representation and working memory with bilinguals. Journal of Communication Disorders, 36 (3), 233-240.

Bachman, L.F. \& Palmer, A.S. (1989). The construct validation of self-ratings of communicative language ability. Language Testing, 6 (1), 14-29. 
Baddeley, A. (2000). The episodic buffer: A new component of working memory? Trends in Cognitive Sciences, 4 (11), 417-423.

Baddeley, A. (2003). Working memory: Looking back and looking forward. Nature Reviews Neuroscience, 4 (10), 829-839.

Baddeley, A.D. \& Hitch, G. (1974). Working memory. Psychology of Learning and Motivation, 8, 47-89.

Baddeley, A.D., Emslie, H., \& Nimmo-Smith, I. (1992). The Speed and Capacity of Language Processing Test. Bury St Edmunds: Thames Valley Test Company.

Baddeley, A., Papagno, C., \& Vallar, G. (1988). When long-term learning depends on short-term storage. fournal Of Memory And Language, 27 (5), 586-595.

Bays, P.M. \& Husain, M. (2008). Dynamic shifts of limited working memory resources in human vision. Science, 321 (5890), 851-854.

Boutla, M., Supalla, T., Newport, E.L., \& Bavelier, D. (2004). Short-term memory span: Insights from sign language. Nature Neuroscience, 7 (9), 997-1002.

Broselow, E. \& Finer, D. (1991). Parameter setting in second language phonology and syntax. Second Language Research, 7 (1), 35-59.

Capirci, O., Cattani, A., Rossini, P., \& Volterra, V. (1998). Teaching sign language to hearing children as a possible factor in cognitive enhancement. Fournal of Deaf Studies and Deaf Education, 3 (2), 135-142.

Carson, J.E., Carrell, P.L., Silberstein, S., Kroll, B., \& Kuehn, P.A. (1990). Reading-writing relationships in first and second language. TESOL Quarterly, 24 (2), 245-266.

Conway, C.M., Pisoni, D.B., \& Kronenberger, W.G. (2009). The importance of sound for cognitive sequencing abilities: The auditory scaffolding hypothesis. Current Directions in Psychological Science, 18 (5), 275-279.

Cowan, N. (2001). The magical number 4 in short-term memory: A reconsideration of mental storage capacity. Behavioral and Brain Sciences, 24 (1), 87-114.

Cummins, J. (1991). Interdependence of first- and second-language proficiency in bilingual children. In E. Bialystok (Ed.), Language Processing in Bilingual Children (pp. 70-89). New York: Cambridge University Press.

Daneman, M. \& Carpenter, P.A. (1980). Individual differences in working memory and reading. Journal of Verbal Learning and Verbal Behavior, 19 (4), 450-466.

Daneman, M. \& Merikle, P.M. (1996). Working memory and language comprehension: A meta-analysis. Psychonomic Bulletin \& Review, 3 (4), 422-433.

Dunn, L.M. \& Dunn, L.M. (1997). Examiner's Manual for the PPVT-III Peabody Picture Vocabulary Test - Third Edition. Circle Pines, MN: American Guidance Service.

Emmorey, K., Kosslyn, S.M., \& Bellugi, U. (1993). Visual imagery and visualspatial language: Enhanced imagery abilities in deaf and hearing ASL signers. Cognition, 46 (2), 139-181.

Emmorey, K., Luk, G., Pyers, J.E., \& Bialystok, E. (2008). The source of enhanced cognitive control in bilinguals evidence from bimodal bilinguals. Psychological Science, 19 (12), 1201-1206. 
Emmorey, K., Borinstein, H.B., Thompson, R., \& Gollan, T.H. (2008). Bimodal bilingualism. Bilingualism: Language and Cognition, 11 (1), 43-61.

Gathercole, S.E. \& Baddeley, A.D. (1989). Evaluation of the role of phonological STM in the development of vocabulary in children: A longitudinal study. Fournal of Memory and Language, 28 (2), 200-213.

Gathercole, S.E. \& Baddeley, A.D. (1990). Phonological memory deficits in language disordered children: Is there a causal connection? Fournal of Memory and Language, 29 (3), 336-360.

Hall, M.L. \& Bavelier, D. (2010). Working memory, deafness, and sign language. In M. Marschark \& P.E. Spencer (Eds.), The Oxford Handbook of Deaf Studies, Language, and Education. Volume 2 (pp. 458-472). New York: Oxford University Press.

Hauser, P., Paludneviciene, R., Supalla, T., \& Bavelier, D. (2006). American sign language - Sentence reproduction test: Development \& implications. RIT Scholar Works, 2006, 155-167.

Hilger, A.I., Loucks, T.M., Quinto-Pozos, D., \& Dye, M.W. (2015). Second language acquisition across modalities: Production variability in adult L2 learners of American Sign Language. Second Language Research, 31 (3), 375-388.

Hirshorn, E.A., Fernandez, N.M., \& Bavelier, D. (2012). Routes to short-term memory indexing: Lessons from deaf native users of American Sign Language. Cognitive Neuropsychology, 29 (1-2), 85-103.

Keehner, M. \& Gathercole, S.E. (2007). Cognitive adaptations arising from nonnative experience of sign language in hearing adults. Memory \& Cognition, 35 (4), 752-761.

MacIntyre, P.D., Noels, K.A., \& Clément, R. (1997). Biases in self-ratings of second language proficiency: The role of language anxiety. Language Learning, 47 (2), 265-287.

Mayberry, R.I. \& Eichen, E.B. (1991). The long-lasting advantage of learning sign language in childhood: Another look at the critical period for language acquisition. Journal of Memory and Language, 30 (4), 486-512.

Miller, G.A. (1994). The magical number seven, plus or minus two: Some limits on our capacity for processing information. Psychological Review, 101 (2), 343-352.

Miyake, A. \& Friedman, N.P. (1998). Individual differences in second language proficiency: Working memory as language aptitude. In A.F. Healy \& L.E. Bourne (Eds.), Foreign Language Learning: Psycholinguistics studies on Training and Retention (pp. 339-364). Mahwah, NJ: Lawrence Erlbaum.

Morere, D. (2008). The Fingerspelling Test. Washington, DC: Science of Learning Institute Visual Language and Visual Learning.

Morere, D., \& Allen, T. (2012). Assessing Literacy in Deaf Individuals: Neurocognitive Measurement and Predictors. New York: Springer.

Oakhill, J. \& Kyle, F. (2000). The relation between phonological awareness and working memory. Journal of Experimental Child Psychology, 75 (2), 152-164. 
Osaka, M. \& Osaka, N. (1992). Language-independent working memory as measured by Japanese and English reading span tests. Bulletin of the Psychonomic Society, 30 (4), 287-289.

Osaka, M., Osaka, N., \& Groner, R. (1993). Language-independent working memory: Evidence from German and French reading span tests. Bulletin of the Psychonomic Society, 31 (2), 117-118.

Padilla, E.R., Lugo, D.E., \& Dunn, L.M. (1986). Test de Vocabulario en Imágenes Peabody (TVIP). Circle Pines, MN: AGS.

Papagno, C. \& Vallar, G. (1995). Verbal short-term memory and vocabulary learning in polyglots. The Quarterly fournal of Experimental Psychology, 48 (1), 98-107.

Park, D.C., Lautenschlager, G., Hedden, T., Davidson, N.S., Smith, A.D., \& Smith, P.K. (2002). Models of visuospatial and verbal memory across the adult life span. Psychology and Aging, 17 (2), 299-320.

Pisoni, D.B., Manous, L.M., \& Dedina, M.J. (1987). Comprehension of natural and synthetic speech: Effects of predictability on the verification of sentences controlled for intelligibility. Computer Speech \& Language, 2 (3), 303-320.

Rosen, V.M. \& Engle, R.W. (1998). Working memory capacity and suppression. fournal of Memory and Language, 39 (3), 418-436.

Saito, S. (2001). The phonological loop and memory for rhythms: An individual differences approach. Memory, 9(4-6), 313-322.

Vallar, G. \& Papagno, C. (2002). Neuropsychological impairments of short-term memory. In A.D. Baddeley, M.D. Kopelman, \& B.A. Wilson (Eds.), Handbook of Memory Disorders (pp. 249-270). Chichester: Wiley.

Vogel, E.K. \& Machizawa, M.G. (2004). Neural activity predicts individual differences in visual working memory capacity. Nature, 428 (6984), 748-751.

Williams, J.T. \& Newman, S.D. (2015, in press). Interlanguage dynamics and lexical networks in nonnative L2 signers of ASL: Cross-modal rhyme priming. Bilingualism: Language and Cognition, 1-18.

Wilson, M. \& Emmorey, K. (1997). A visuospatial "phonological loop" in working memory: Evidence from American Sign Language. Memory \& Cognition, 25 (3), 313-320.

Wilson, M. \& Emmorey, K. (1997). Working memory for sign language: A window into the architecture of the working memory system. Fournal of Deaf Studies and Deaf Education, 2 (3), 121-130.

Wilson, M. \& Emmorey, K. (1998). A "word length effect" for sign language: Further evidence for the role of language in structuring working memory. Memory \& Cognition, 26 (3), 584-590.

Wilson, M., Bettger, J.G., Niculae, I., \& Klima, E.S. (1997). Modality of language shapes working memory: Evidence from digit span and spatial span in ASL signers. Journal of Deaf Studies and Deaf Education, 2 (3), 150-160. 